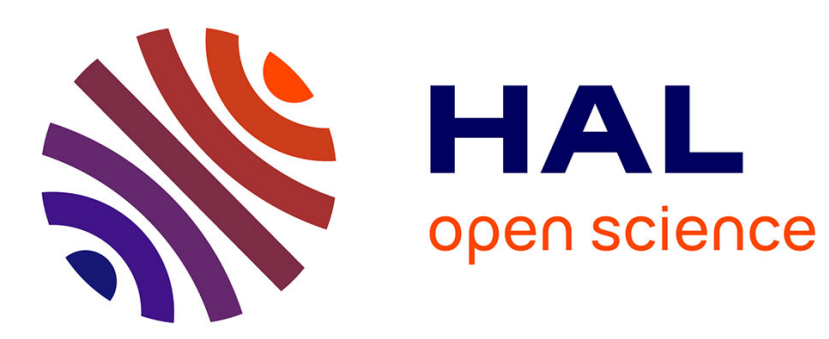

\title{
Diagrams, gestures and formulae in music
}

Guerino Mazzola, Moreno Andreatta

\section{To cite this version:}

Guerino Mazzola, Moreno Andreatta. Diagrams, gestures and formulae in music. Journal of Mathematics and Music, 2007, 1 (1), pp.23-46. hal-01161060

\section{HAL Id: hal-01161060 \\ https://hal.science/hal-01161060}

Submitted on 8 Jun 2015

HAL is a multi-disciplinary open access archive for the deposit and dissemination of scientific research documents, whether they are published or not. The documents may come from teaching and research institutions in France or abroad, or from public or private research centers.
L'archive ouverte pluridisciplinaire HAL, est destinée au dépôt et à la diffusion de documents scientifiques de niveau recherche, publiés ou non, émanant des établissements d'enseignement et de recherche français ou étrangers, des laboratoires publics ou privés. 
This article was downloaded by:[Andreatta, Moreno]

On: 26 March 2007

[Andreatta, Moreno]

Access Details: Sample Issue Voucher: J ournal of Mathematics and Music [subscription number 775682644]

Publisher: Taylor \& Francis

Informa Ltd Registered in England and Wales Registered Number: 1072954

Registered office: Mortimer House, 37-41 Mortimer Street, London W1T 3J H, UK

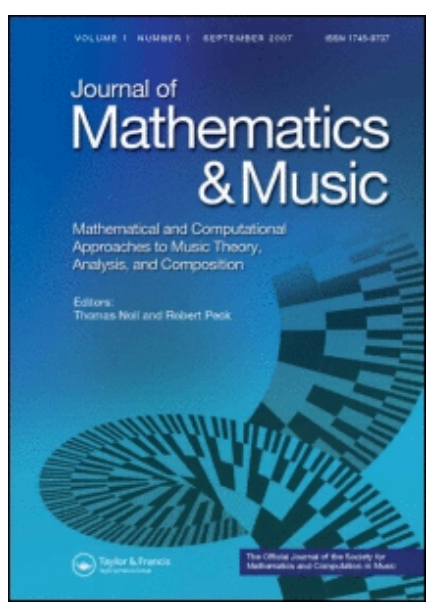

\section{J ournal of Mathematics and Music Mathematical and Computational Approaches to Music Theory, Analysis, Composition and Performance}

Publication details, including instructions for authors and subscription information: http://www.informaworld.com/smpp/title content=t741809807

Diagrams, gestures and formulae in music

To cite this Article: , 'Diagrams, gestures and formulae in music', J ournal of Mathematics and Music, $1: 1,23-46$

xxxx:journal To link to this article: DOI: $10.1080 / 17459730601137716$

URL: http://dx.doi.org/10.1080/17459730601137716

Full terms and conditions of use: http://www.informaworld.com/terms-and-conditions-of-access.pdf

This article maybe used for research, teaching and private study purposes. Any substantial or systematic reproduction, re-distribution, re-selling, loan or sub-licensing, systematic supply or distribution in any form to anyone is expressly forbidden.

The publisher does not give any warranty express or implied or make any representation that the contents will be complete or accurate or up to date. The accuracy of any instructions, formulae and drug doses should be independently verified with primary sources. The publisher shall not be liable for any loss, actions, claims, proceedings, demand or costs or damages whatsoever or howsoever caused arising directly or indirectly in connection with or arising out of the use of this material.

(c) Taylor and Francis 2007 


\title{
Diagrams, gestures and formulae in music
}

\author{
GUERINO MAZZOLA*† and MORENO ANDREATTA**†
}

$\dagger$ Institut für Informatik, Universität Zürich, Binzmühlestrasse 14, CHC-8050 Zürich, Switzerland

†Equipe Représentations Musicales, IRCAM/CNRS, UMR 9912, 1 Place I. Stavinsky, F-75004, Paris, France

(Received 21 May 2006; in final form 10 October 2006)

\begin{abstract}
This paper shows an interplay of music and mathematics which strongly differs from the usual scheme reducing mathematics to a toolbox of formal models for music. Using the topos of directed graphs as a common base category, we develop a comprising framework for mathematical music theory, which ramifies into an algebraic and a topological branch. Whereas the algebraic component comprises the universe of formulae, transformations, and functional constraints as they are described by functorial diagrammatic limits, the topological branch covers the continuous aspects of the creative dynamics of musical gestures and their multilayered articulation. These two branches unfold in a surprisingly parallel manner, although the concrete structures (homotopy versus representation theory) are fairly heterogeneous. However, the unity of the underlying musical substance suggests that these two apparently divergent strategies should find a common point of unification, an idea that we describe in terms of a conjectural diamond of categories which suggests a number of unification points. In particular, the passage from the topological to the algebraic branch is achieved by the idea of the gestoid, an 'algebraic' category associated with the fundamental groupoid of a gesture.
\end{abstract}

Keywords: Gesture; Fundamental group; Formulae; Networks; Spectroids

\section{Introduction}

This paper presents a new programmatic category-oriented framework for the description of the relations between musical and mathematical activities. This relation may be described in terms of adjointness between functors, which extend the functorial setup discussed in The Topos of Music [1]. Thus, on a meta-level, the relations between musical and mathematical activities are investigated from a mathematical point of view.

Far from being isomorphic, music and mathematics seem to involve some common structures that can be related by one of the most powerful concepts of category theory: the notion of adjoint functors. This construction, proposed by Daniel Kan in the fifties

\footnotetext{
*Corresponding author. Email: guerino@mazzola.ch

**Email: Moreno.Andreatta@ircam.fr
} 
as a technical device for the study of combinatorial properties in homotopy theory [2], turns out to be the most adequate tool to link three main categories: equations or formulae (category of spectroids), diagram schemes (category of directed graphs) and gestures (category of diagrams of curves in topological spaces).

The category of directed graphs, which has been recently proposed as a foundational concept in mathematics for both classical and categorical set-theory [3], seems to provide a musically interesting mediating structure between the two other categories, on which music and mathematics act in adjoint positions. By means of diagrams, mathematics turns gestures into formulae. In fact, a diagram is a system of transformational arrows. On such a system you may follow different paths starting and ending at the same two points. These paths can be viewed as gestural movements. If two such paths commute, i.e. they yield the same composed transformation, then we have exactly what is called a formula or equation: two expressions yield the same result. Quite generally speaking, formulae are commutativity relations between gestural paths. Conversely, musical activity 'unfreezes' formulae into gestures that can be described as the unfolding of formulae in space-time.

Within the conceptual framework of this paper we want to include embodied performance into the formalization of musical structures. In our recent paper [4] we argued that the categorical presentation of Klumpenhouwer networks as elements in limits of diagrams of spaces and transformations has some important operational consequences. A parallel argument applies to the use of the category of directed graphs of curves in topological spaces as a theoretical framework for gesture theory. From a purely theoretical aspect, 'gestures of gestures' (or hypergestures) as well as 'natural gestures' are canonically defined, as we will see by discussing the case of the gesture of a finger of a piano player's hand and its hypergestural generalizations. Similar to the case of the development of category and topos theory, as discussed by Mac Lane in [5], the notion of gesture as suggested in the present paper offers a good illustration of the 'collision' between algebraic and topological methods.

But there is another intriguing aspect of this new categorical setup for musical gestures that we would like to mention too, and this deals with the philosophical ramifications of category theory. Category theory is more than a useful universal language, eventually providing the theoretical setting for the foundations of mathematics. When applied to a complex human activity such as music, category theory offers the conceptual framework generating a new theoretical perspective of the relations between the philosophy of music and the philosophy of mathematics, in fact, by shedding new light on the understanding of the genesis and ontology of musical and mathematical activities.*

Moreover, as suggested by the recent developments of Jean Piaget's genetic epistemology [9, p. 217], 'la théorie des catégories, considérée comme théorie des constructions mathématiques, reflète la constitution génétique des outils cognitifs de l'homme: le détachement des schèmes transférables d'un ensemble d'actions, puis des

\footnotetext{
* Essentially, this is due to Alexander Grothendieck's reinvention of the point concept in algebraic geometry. He redefines a point as being a morphism $f: X \rightarrow Y$ in the category of schemes, and conversely, using the Yoneda lemma, a morphism $f: X \rightarrow Y$ in any category can be viewed as a point in the presheaf associated with $Y$. This means that the original Euclidean point concept (punctus est cuius pars nulla est) is replaced by the elementary concept of a morphism, which determines the ontology of a category. This point of view suggests that such morphisms, which are commonly understood as 'arrows', induce a dynamical aspect: a morphism is the movement of an arrow. In this spirit, mathematical activities are presently being debated as gestural movements along such arrows, instead of abstract manipulation of symbols [6]. But the arroworiented approach to mathematics also enables a description of basic musical concepts as activities in terms of morphisms. David Lewin's transformational theory [7], Thomas Noll's harmonic morphology [8] or our categorical approach to performance theory [1, chapter 35] are examples thereof.
} 
opérations semblables sur ces schèmes, puis sur des schèmes de schèmes et ainsi de suite.' It is remarkable that in the study of $n$-dimensional space-time, category theory has become a key paradigm in physics since 1990. As in genetic epistemology, it is also the iterated process construction (in terms of $n$-categories) that comes into play for physics. Let us complete the picture with the remark that iterated processes are also central in transformational theory, and therefore can be elegantly conceptualized in a categorical perspective, see [4].

The case of gesture theory suggests that we can naturally transfer Mac Lane's conception of mathematics as 'an elaborate tightly connected network of formal systems, axiom systems, rules, and connections' [10, p. 417] to music. The adjoint functors that we establish between the formal category of formulae and the functional universe of gestures suggests that musical activity could also be conceived as arising from a formal network based on some dynamic concepts that evolve according to their function. This framework has some very interesting philosophical consequences, especially when trying to update the debate on the relation between the structural conception of mathematics and the structuralist approach to music.* As in the case of the discussion between mathematics and structuralism, for which category theory has played a major role $[3,12,13]$, the account of a structuralist position in music-theoretical research could greatly benefit from a categorical approach.

The paper suggests that mathematical structuralism could be taken as a philosophical position for music-theoretical activity once it is accepted that mathematical music theory is about music conceived as a structured system. As rightly observed by Elaine Landry and Jean-Pierre Marquis in an interesting attempt at putting category theory into an historical, foundational and philosophical context [12], 'the problem with standard structural approaches is that they cleave to the residual Fregean assumption that there is one unique context that provides us with the pre-conditions for the actual existence of 'structures' or for the possible existence of types of structured systems' [12]. And as the categorical framework suggests that 'mathematical concepts have to be thought of in a context that can be varied in a systematic fashion' [12, p. 21], the categories of formulae, diagrams, and gestures in music suggest that the functorial adjointness that we shall present provides a general framework for the study of gestures in a given musical context.

Unfolded from the scant category of digraphs, the categories of linear categories associated with digraphs (spectroids [14]) and the categories of gestures split the structural content of the morphism concept: whereas the algebraic context of spectroids (which also includes the transformational approach to music theory) leaves the morphism concept in its abstract setup inherited from the classical Fregean approach to functions, i.e. the totally abstract relation between argument and functional value, the category of gestures is built upon morphisms that are derived from continuous curves, such that the movement from argument to value is mediated along the entire curve following the curve parameter. The gesture is a morphism, where the linkage is a real movement and not only a symbolic arrow without bridging substance. The arrow is a symbol of category theory that suggests a bridge between domain and codomain and thereby points to a metaphor overloaded by embodiment. However, according to Jean Cavaillès [15] 'Comprendre est attraper le geste et pouvoir continuer.' This means that

\footnotetext{
* See [11] for a detailed discussion on the emergence and rise of the notion of mathematical structure in music from an algebraic perspective.
} 
human evidence and operational competence are intimately tied to the embodied movement, and this is a gesture, not the abstract arrow.

This paper therefore argues that the gestural movement should be the missing link between abstract formulaic intelligence and plain bodily gesticulation. And it offers a generative force in the creation of significance and the regularity of rule-based systems. This refers to a substantial insight of Charles Alunni [6]: 'Ce n'est pas la règle qui gouverne l'action diagrammatique, mais l'action qui fait émerger la règle.'

The plan of the paper is as follows. Sections $2-6$ deal with the gestural branch of the theory - in Section 2, we give a short account of the notion and significance of gestures in music and thereby suggest that gestures could play the same role in music as strings in particle physics. We then (Section 3) introduce the mathematical category of gestures in terms of diagrams of directed graphs (digraphs) in topological spaces. In Section 4, we show that the space of gestures in a given topological space is a topological one. Therefore the iteration of the gesture constructions is possible and naturally leads to the notion of hypergestures. In Section 5, we prove the existence of a 'natural' gesture associated with a digraph and then the adjunction of this functor with the functor projecting a gesture to the underlying digraph. Using techniques from homotopy theory and linear category theory, we introduce the notion of the linear gestoid category associated with a gesture in Section 6 and then (Section 6.1) we conjecture a relation between K-nets and gestures that uses the gestoid construction. This section terminates with an interesting interpretation of the gestoid of a circular gesture in terms of the Fourier representation of complex sounds.

Sections 7-8 deal with the formulaic branch of the theory: Section 7 provides an algebraic interpretation of abstract digraphs in terms of linear categories (spectroids) associated with the path category of a digraph, as introduced by Peter Gabriel. This includes the study of local networks as solutions of 'formulae', which are conceived as commutativity conditions on diagrams of modules. In this context, 'formulae' play the analogous role of gestures. Here, the previous topological presentation of gesture spaces is replaced by the algebraic presentation using the radical of a spectroid. We then prove the analogous adjunction theorem for natural formulae instead of natural gestures. By analogy with the gestoid construction on the topological branch of our discussion, we then introduce the tangent category of a formula and then the associated radical formoid in Section 8. For some digraphs, the gestoids of the associated natural gestures and also radical formoids of the associated natural formulae are calculated.

Sections 9-11 deal with the unification of both branches and the outlook. In Section 9, we depict the diagram of the previously discussed categories and functors with their adjunction pairing. The diagram is completed by a conjectural ideal category $X$, providing a unifying diamond diagram for the topological and algebraic branches of the main categories in music. The future implementational aspects of the theory discussed in this paper are briefly exposed in Section 10. In the final Section 11, we draw some conclusions and give suggestions for future research, in particular regarding the gestural logic, which is provided by the canonical topos logic of the category of directed graphs.

\section{Gestures: musical string theory}

It is well known that the formal description, classification, and analysis of music in terms of local or global systems of note sets in adequate parameter spaces does not grasp the full reality of music. 
In fact, beyond this music-theoretical reality, music must be performed, i.e. the formally parametrized note configurations must be mapped into physically meaningful spaces - see [16] for a musicological account of performance theory and [1, parts XIII, IX] for a more mathematical and computational overview. Although performance theory may yield sophisticated sounds from a 'mechanical' score, it does not embody the musician's instrumental activity. When we play music, we make the performed sounds. This is more than a set of sound events, more than a CD recording may ever trace on its acoustic level. The important role of embodiment of sounds is rightly testified by the strong need for concerts, where performance is not only heard, but also experienced from the musicians' bodies in movement. Understanding music is strongly enhanced if not enabled by means of its presentation in moving bodies, or, to put it more concisely, in musical gestures.

It may be argued that gestures are, like performance of shaped sounds, rhetorical means to convey a meaning that in principle is faithfully represented in the score's content. This is however erroneous for two reasons. To begin with, score signs are not unambiguously loaded with meaning. The creation of signification of musical signs shares a deictic nature. Only the user (in particular, the performing artist) can complete the partial meaning of musical score signs. It can be shown that there is an infinity of such completions, be it on the symbolic level [1, chapter 13.4.1], or be it on the level of agogics, articulation or dynamics [1, chapter 45]. The second - really dramatic - reason is that there are many types of music that are not subjected to the scheme 'score to performance', at least not if score means a structural or processual scheme controlling sound production. Much jazz music, for example, is defined through its bodily realization rather than as a projection of score information. Cecil Taylor's piano music is a stunning example of the primacy of bodily gestures (what Volker Spicker calls the 'Abstraktmotiv' [17]) over structures, a fact often pointed out by Taylor himself. And already for Beethoven, as also observed by Robert Hatten [18], ruling out gestural shaping misses the musical contents, as beautifully illustrated by Glenn Gould's notorious 'contrafactual' recording of Op. 57 'Appassionata', where, for example, the cascade in bars 14-15 of the opening movement is not played as a gestural cascade, but as a static structure of arpeggiated VII-chords, which destroys completely the inherent movement, as performed by Vladimir Horowitz, say.

Despite the intuitive understanding of what is a gesture, including body movement and semantics, a precise conceptualization looks less easy. We agree with Jean-Claude Schmitt [19] that the medieval definition of a gesture, as given by Hugues de SaintVictor, remains one of the most adequate, at least when referring to the concrete human body: 'Gestus est motus et figuratio membrorum corporis, ad omnem agendi et habendi modum.' Gesture is the movement and figuration of the body's limbs with an aim, but also according to the measure and modality proper to the achievement of all action and attitude.* Most important is that it is an articulated figuration, a composition of parts (limbs), and that it includes a movement of that figuration in the space-time of the given body. Moreover, it serves for any (omnem) mode of action and attitude, so it has a purpose or target, but it does not, automatically, point to a semantic level, it only reaches the mode of an activity/habit. ${ }^{\dagger}$ Often, in the definition of a gesture, the sign

\footnotetext{
* We follow the English translation by Roman Katsman [20], see also http://www.utoronto.ca/tsq/12/ katsman12.shtml.

$\dagger$ This is also confirmed by Jean-Claude Schmitt [19] in his comment on Saint-Victor's definition: 'Le geste enveloppe avant de saisir et esquisse son déploiement bien avant de dénoter ou d'exemplifier; ce sont les gestes déjà domestiqués qui font référence.'
} 
character (signifier-signification-signified) is included, for example in David McNeill's approach [21]. Together with the French diagrammatic philosophers, such as Charles Alunni [6], we do not share this perspective and would rather say that a gesture is a presemiotic entity, although it may be a component of a sign. But this is not mandatory. For example, in dance or in music, gestures are often bare of meaning. They stand for themselves, and this in particular as aesthetic entities. We should also add that our reference to Saint-Victor does not mean that we shall limit ourselves to this definition. It is only a point of departure, and we shall transcend its strict reference to the human body and its limbs and consider much more abstract or metaphoric configurations in the following mathematical theory of gestures, including gestural objects relating to graphics and sounds. But what is important for our understanding of the concept is that in Saint-Victor's definition, the composed parts on the one hand and the presemiotic nature of a gesture on the other are recognized.

The shift from abstract algebra and transformational paradigms to gestures is not a purely formal one, since the basic object given by a gesture is not a 'point' within an algebraically shaped space, which may be connected to other points by functional correspondence. A gesture, by its parametrized curve character, has interiorized the transformational approach: its endpoints are intrinsically related by the curve parameter. This is in contrast to the functional correspondence, where an arrow effectively has nothing that exists in its 'middle', it is a purely graphical symbol. No additional transformational input is needed.

This is an enrichment that has been realized for similar constructions in the physical string theory of elementary particles. In this language, particles are also curves of an ample inner nature, when compared to the classical point-like elementary particle models. For example, in string theory, the electric charge of an electron may be constructed geometrically as a winding number of the electron loop around a supplementary compact dimension, i.e. by an instance of the fundamental group of the compact dimension. In this spirit, the gestural approach is an enrichment of musical object categories, which enables a refinement of the conceptual anatomy and at the same time a rapprochement to the human reality of making music. We could call it a musical string theory.

\section{Digraphs and gestures}

In the following definition of a gesture, we shall rely on Saint-Victor's definition and implement the (con)figuration of a gesture by the articulation of diagrams. For an approach to the concept of coordinated gestures, which uses diagrams and their limits; see Noll's paper [22]. We shall then describe the movement in the parametrization of curves representing the figuration, and we shall formalize the body's space-time by a topological space, where the movement takes place. The semantics of gestures will not be our concern here; this must be dealt with after a thorough investigation of the formal mathematics of gestures.

Let us first review the category Digraph of directed graphs, in short-digraphs. This is a basic category for algebra as well as for topology. In the naive setup, its objects are functions $\Gamma: A \rightarrow V^{2}$ from a set $A=A_{\Gamma}$ of arrows to the Cartesian square $V^{2}=V \times V$ of the set $V=V_{\Gamma}$ of vertices. The first projection $t=p r_{1} \circ \Gamma$ is called the tail function, the second $t=p r_{2} \circ \Gamma$ is called the head function of the digraph. For an arrow $a$, the vertices $t(a), h(a)$ are called its head and tail, respectively, and denoted by $t(a) \stackrel{a}{\rightarrow} h(a)$. 
A morphism $f: \Gamma \rightarrow \Delta$ of digraphs is a couple $f=(u, v)$ of functions $u: A_{\Gamma} \rightarrow A_{\Delta}, v: V_{\Gamma} \rightarrow$ $V_{\Delta}$ such that $v^{2} \circ \Gamma=\Delta \circ u$.

A more creative definition of this category is to view it as the category of presheaves* $h t^{\mathrm{a}}$ over the small category $h t=\overbrace{t}^{h}$. with two objects and just two parallel morphisms $h, t$ between these points. This interpretation ${ }^{\dagger}$ implies that Digraph is a topos. In particular, it has a final object $1=t T$, which is embedded by the true morphism $T: 1 \rightarrow \Omega$ into a subobject classifier

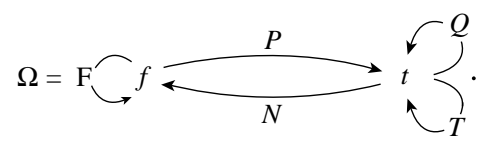

Every evaluation $\Gamma @ \Omega$ describes the set of subdigraphs of $\Gamma$, together with its canonical Heyting logic. This fact may be used to introduce gestural logic; see our final remark in Section 11.

In this paper, we need a special subcategory of digraphs, the spatial digraphs. Such a digraph is associated with a topological space $X$ and denoted by $\vec{X}$. By definition, the arrow set is $A_{\vec{X}}=I @ X$, the set of continuous curves $c: I=[0,1] \rightarrow X$ in $X$, while the vertex set is $V_{\vec{X}}=X, h(c)=c(1)$, and $t(c)=c(0)$. A spatial morphism is a digraph morphism $\vec{f}: \vec{X} \rightarrow \vec{Y}$ canonically induced by a continuous map $f: X \rightarrow Y$. The subcategory of spatial digraphs and morphisms is denoted by SpaceDigraph. A spatial digraph is more than a digraph: it is also a topological digraph in the following sense. The set $A_{\vec{X}}=I @ X$ of arrows of $\vec{X}$ is a topological space by the compact-open topology, and the head and tail maps $h, t: I @ X \rightarrow X$ are continuous. Moreover, for a continuous map $f: X \rightarrow Y$, the arrow map $I @ f: I @ X \rightarrow I @ Y$ is continuous.

Given a digraph $\Delta$ and a topological space $X$, a $\Delta$-gesture in $X$ is a digraph morphism $\delta: \Delta \rightarrow \vec{X}$, i.e. a realization of the abstract vertices and arrows within a topological space, as shown in figure 1 . It is essential here to distinguish the curve parameter of a gesture from the time parameter, which intervenes in a number of common gestures. For example, when drawing a curve on a sheet of paper, this gesture $\delta: \Delta \rightarrow \vec{X}$ would have the arrow digraph $\Delta=\uparrow$ with $\uparrow=\bullet \rightarrow \bullet$ and the space $X=\mathbb{R}^{2} \times \mathbb{R}$, whose first two coordinates denote the points of the paper surface, whereas the third denotes the physical time when a point at given parameter value is drawn on the paper sheet. We discuss the time parameter in Example 3.1 below.

Given two gestures $\delta: \Delta \rightarrow \vec{X}, \gamma: \Gamma \rightarrow \vec{Y}$, a morphism $f: \delta \rightarrow \gamma$ is a digraph morphism $f: \Delta \rightarrow \Gamma$ such that there exists a spatial morphism $\vec{h}: \vec{X} \rightarrow \vec{Y}$ that commutes with $f$, i.e. $\vec{h} \circ \delta=\gamma \circ f$. This defines the category Gesture of gestures, and we have a projection $p$ : Gesture $\rightarrow$ Digraph, which sends the gesture $\delta: \Delta \rightarrow \vec{X}$ to the underlying digraph $\Delta$. So it is essentially a forgetful functor: it cancels out the spatial interpretation of the given digraph.

Example 3.1 Let us give here an elementary example of the gesture of a finger of a piano player's hand. The gesture represents the movement of a finger going down to a key, keeping in that position for the duration of a tone, and then moving back upwards

\footnotetext{
${ }^{*}$ For any category $\mathcal{C}, \mathcal{C}^{@}$ denotes the category of presheaves, i.e. contravariant set-valued functors, on $\mathcal{C}$. Our notation stems from the Yoneda embedding $y: \mathcal{C} \rightarrow \mathcal{C}^{@}$, which associates with an object $X$ in $\mathcal{C}$ the presheaf@X which yields the set@ $X(Y)=Y @ X$ of morphisms $f: Y \rightarrow X$ in $\mathcal{C}$.

$\dagger$ Observe that $h t^{o p} \leadsto h t$, so it is also legitimate to view $h t^{@}$ as being built from the covariant functors on $h t$.
} 


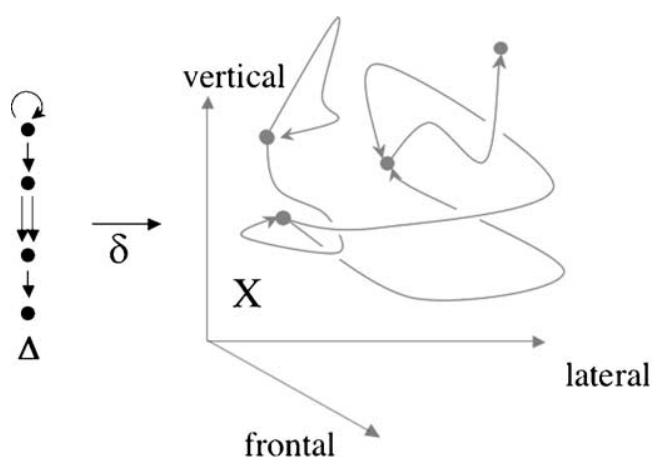

Figure 1. A gesture in the ordinary 3-space.

to be ready for a next movement. This gesture has three segments, which we formally

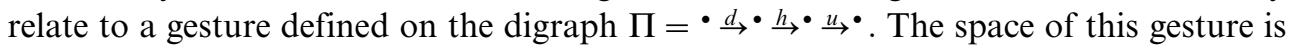
three-dimensional, i.e. FingerSpace $=\mathbb{R}^{3}$, where the first is the height above the keyboard (position, positive direction for approaching the piano), the second is the time, and the third coordinate parametrizes the pitch (the coordinate of the key on the piano's keyboard). Then a finger gesture $\pi: \Pi \rightarrow \overrightarrow{\text { FingerSpace }}$ is a diagram of curves as shown in figure 2.

Example 3.2 A more involved example is provided by the gestural space of an entire hand, as investigated in [23] in the context of the simulation of a pianist's playing from a given score, under constraints from the hand's geometry as well as the physical dynamics from Newton's second law (limits of forces avaliable from the pianist's performance). Figure 3 shows the hand position in time. It is given by six points $\gamma_{\mathrm{i}}(t)$ in the 4-space $\mathbb{R}^{4}$ with three space coordinates (pitch, vertical position above keyboard, and horizontal frontal position from the key face line) and one time coordinate. The finger tips are represented by the indexes $i=1,2, \ldots, 5$, while the carp is represented by $i=6$. Observe that the time coordinate is not the curve coordinate $t$ ! A gesture's curve coordinate is an abstract parametrization of the curve in a given space, not the material time coordinate, which may also be absent, as shown in the dance gesture from figure 6.

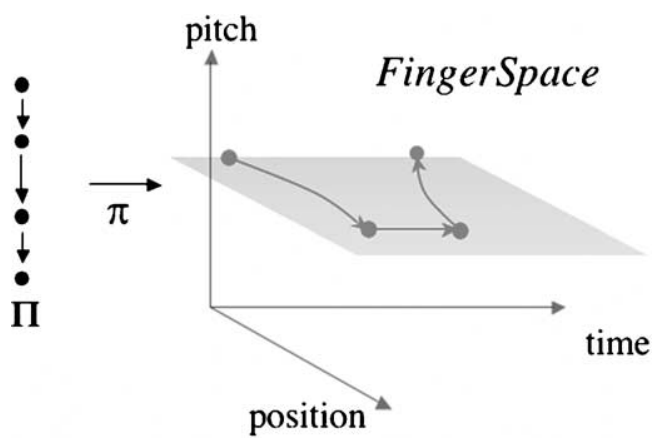

Figure 2. An elementary finger gesture of a pianist's hand. 


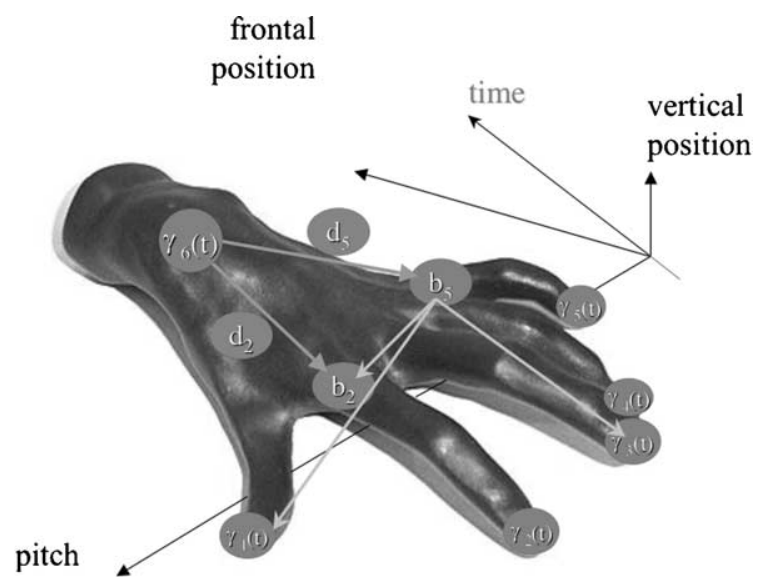

Figure 3. The coordinates of a pianist's hand for its representation in a gesture space.

\section{Hypergestures}

If gestures of a certain kind are themselves conceived as points in a space, one may study gestures within such a gesture-space. These may be called hypergestures. In order to define them, we need to know how to turn the set of gestures $\Delta @ \vec{X}$ into a topological space. Now, the special case $\Delta=\uparrow$ means that we have the topological space $\uparrow @ \vec{X} \leadsto I @ X$ of continuous curves $c: I \rightarrow X$ (with the above mentioned compact-open topology). The general case follows from the observation that $\Delta$ is the colimit of the following diagram $\mathcal{D}$ of digraphs: we take one arrow digraph $\uparrow_{a}=\uparrow$ for each arrow $a \in A_{\Delta}$ and one bullet digraph $\bullet_{x}=\bullet$ for each vertex $x \in V_{\Delta}$. We take as morphisms the tail or head injections $\bullet_{x} \rightarrow \uparrow_{a}$ whenever $x=t(a)$ or $x=h(a)$. Then evidently, $\Delta \stackrel{\rightarrow}{\rightarrow}$ colim $\mathcal{D}$. Therefore, the set of gestures $\Delta @ \vec{X}$ is bijective to the limit $\lim \mathcal{D} @ \vec{X}$ of a diagram of topological spaces $\uparrow @ \vec{X} \leadsto I @ X$. The topology of $\Delta @ \vec{X}$ is defined as the limit topology of this diagram, the space with this topology is denoted by $\Delta \vec{@} X$. In the case of a metric $d$ defining the topology of $X$, it is well known that the compact-open topology on $I @ X$ coincides with the topology defined by the metric $\vec{d}\left(\gamma, \gamma^{\prime}\right)=$ $\sup _{t} d\left(\gamma(t), \gamma^{\prime}(t)\right)$. And then, the topology of $\Delta \vec{@} X$ is defined by the metric $\left.\Delta \vec{\Theta} d\left(\gamma, \gamma^{\prime}\right)=\Sigma_{a \in A_{\Delta}} \overrightarrow{d(} \gamma_{a}, \gamma_{a}^{\prime}\right)$.

This construction is functorial in both arguments - if $h: \Gamma \rightarrow \Delta, f: X \rightarrow Y$ are a couple of morphisms of digraphs and topological spaces, respectively, then the canonical map $(f, h)=\overrightarrow{f \circ} ? \circ h: \Delta \vec{@} X \rightarrow \Gamma \vec{@} Y$ is continuous and functorial in $h, f$. For example, if $h: p \rightarrow \Delta$ is the embedding of a single vertex $p$, the map $\left(I_{X}, h\right): \Delta \vec{@} X \rightarrow p \vec{@} X$ is the restriction of gestures to the point $p$. If we select a particular gesture $\xi$ in $p \vec{@} X$, i.e. a point $\xi \in X$, then the fibre $\left(I d_{X}, h\right)^{-1}(\xi)$ is the set of gestures sending $p$ to $\xi$. If in particular $\Delta=1$, this fibre is the set of loops in $\xi$.

By the above, one may now repeat the gesture construction and consider the topological (and, especially, the metric) space of hypergestures $\Gamma \vec{@} \Delta \vec{@} X$, hyperhyper-

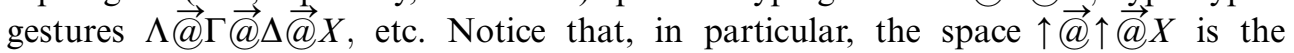
topological space $I^{2} @ X$ of homotopies in $X$, i.e. hypergestures generalize homotopies between continuous curves. There follows a useful proposition concerning the order in which hypergestures are constructed. 
Proposition 4.1 If $\Gamma, \Delta$ are digraphs and $X$ is a topological space, then we have a canonical homeomorphism

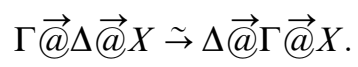

This results from the above fact that a digraph is the colimit of its arrows, glued together on their head and tail points. On the one hand, this implies that the hypergesture space $\Gamma \vec{a} \Delta \vec{@} X$ is the limit of the spaces with arrows $\uparrow \vec{a} \Delta \vec{a} X$, which are in turn identified with the spaces of curves $I @ \Delta \vec{@} X$, but these are the limit of spaces $I @ I @ X$ over the arrow system of $\Delta$. On the other hand, one may also first start with the limit over the arrow system of $\Delta$ and then pass to the limit over the arrow system of $\Gamma$. Thus, the two limit systems can be applied in any order, and this means that the two topological spaces in question are isomorphic by the universal properties of colimits and limits. One may also use the fact that double limits exchange.

COROllary 4.2 The action

$$
\vec{@}: \text { Digraph } \times \text { Top } \rightarrow \text { Top }:(\Gamma, X) \mapsto \Gamma \overleftrightarrow{@} X
$$

canonically extends to an action (denoted by the same symbol)

$$
\vec{a}:[\text { Digraph }] \times \mathbf{T o p} \rightarrow \mathbf{T o p}:(W, X) \mapsto W \vec{a} X
$$

of the free commutative monoid [Digraph], i.e. the monoid of commutative words $W=$ $\Gamma_{1} \Gamma_{2} \ldots \Gamma_{k}$ over the alphabet Digraph of digraphs (the objects only). It is defined* inductively by $\Gamma_{1} \Gamma_{2} \ldots \Gamma_{k} \vec{@} X=\Gamma_{1} \vec{@}\left(\Gamma_{2} \ldots \Gamma_{k} \vec{@} X\right)$ and $\emptyset \vec{@} X=X$.

Example 4.3 Referring to the finger gestures described in Example 3.1, we want to discuss an example of piano finger hypergesture. Before so doing, let us mention a quite intriguing statement of Renate Wieland and Jürgen Uhde in [24]:

Die Klangberührung ist das Ziel der zusammenfassenden Geste, der Anschlag ist sozusagen die Geste in der Geste. ${ }^{\dagger}$

Although the precise meaning of this statement is somewhat cryptic, it could be interpreted as arguing that a gesture may be thought of as being built from other gestures. Recall that a hypergesture is built from a system of homotopies of the curves that compose its vertex gestures. Now, let us take a hypergesture $\eta: \uparrow \rightarrow \overrightarrow{\text { FingerSpace }}$. This means that we have a continuous curve of finger gestures $\eta(t), t \in I$, from the initial finger gesture $\eta(0)$ to the final finger gesture $\eta(1)$.

Musically, this means that in the given FingerSpace, two gestures can be related to each other by thinking of the final gesture as a result of an infinite series of intermediate gestures. So they may be connected cognitively by just deforming the original gesture to obtain the final one. This seems to be obvious in our example, since no obstruction to such a deformation is indicated. The next example shows that in a less trivial space, viz. the torus, such deformations are not always possible and, from a cognitive point of view, this would lead to conceiving radically different strategies in the

\footnotetext{
* To be precise, this action is defined up to homeomorphisms.

$\dagger$ The touch of sound is the target of the embracing gesture, the keystroke is so to speak the gesture within the gesture.
} 
management of gestures. In fact, the existence of hypergestures relates to the fundamental group of the underlying topological space.

Example 4.4 Take the torus $X=T$ and the final digraph 1; see figure 4 . Then, if $\eta, v: 1 \rightarrow \vec{T}$ are the horizontal equatorial circle curve at the origin 0 of $T$ and the vertical meridian curve at the origin, respectively, there is no hypergesture of type $\uparrow$ from $\eta$ to $v$, whereas the diagonal reflection on $T$ yields the morphism $I d_{1}: \eta \rightarrow v$.

This makes clear the difference between hypergestures and morphisms: hypergestures realize the 'arrows' between vertex gestures as curves, whereas morphisms realize them by transformations between the vertex curves.
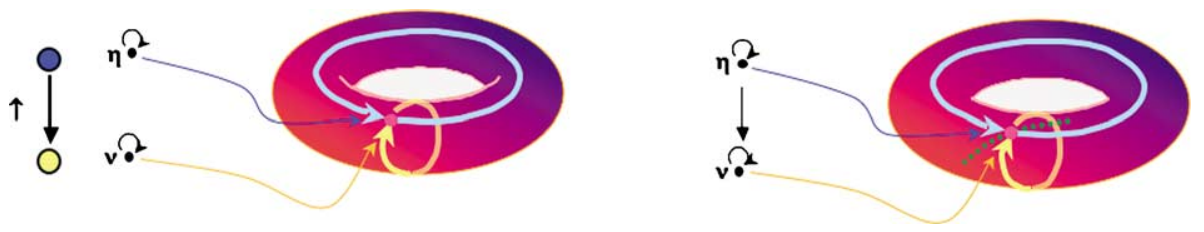

Figure 4. There is no hypergesture from $\eta$ to $v$ (left), while these two gestures are isomorphic (right).

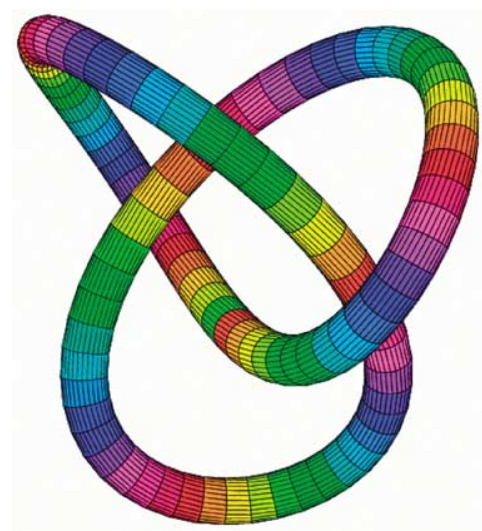

Figure 5. A knot represents a complex hypergesture built from loop gestures.
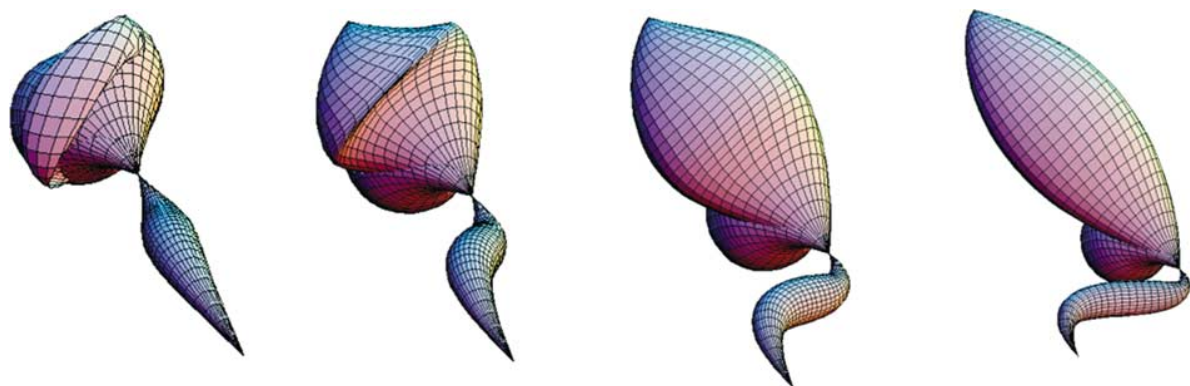

Figure 6. Four stages (from left to right) of a 'dancing' hypergesture of type $\uparrow$ with values in spatial

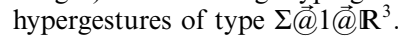




\subsection{Spatial hypergestures}

Intuitively, gestures allude to a movement in space, where the curve parameter plays the role of time. This is however misleading and restrictive. Let us explain it by the representation of complex bodies as spatial hypergestures. If we start with the space of loops in $3 \mathrm{D}$, i.e. the gesture space $X=1 \vec{@} \mathbb{R}^{3}$, a gesture of diagram $\Gamma$ in $X$ is a hypergesture $\beta \in B=\Gamma \vec{@} 1 \vec{@} \mathbb{R}^{3}$; see figure 5 for an example.

With spatial hypergestures, one can essentially do all the computer graphics constructions, such as spline surfaces of Bézier type [25]. Such a surface, when defined by a grid of $(n+1) \times(m+1)$ points appears as a hypergesture $\uparrow^{n} \vec{@} \uparrow^{m} \vec{@}, \mathbb{R}^{3}$, where $\uparrow^{n}$ is the digraph consisting of $n+1$ vertices, and having one arrow from vertex $i$ to vertex $i+1$ for all $i=0,1,2, \ldots, n-1$. Then, using this type of purely spatial hypergesture $\beta$, which we temporarily call bodies, we may model the movement of such a body in time as a gesture $\mu \in \uparrow \overleftrightarrow{@ B} B \times \mathbb{R}$, having values in the space of pairs $(\beta, \tau)$ of bodies and times. This allows for the description of realistic body movements (viz. animated graphics) for dance or sports, for example. In fact, $\uparrow @ \vec{a} B \times \mathbb{R} \sim \uparrow \vec{a} B \times \uparrow \vec{@} \mathbb{R}$, i.e. such a movement is

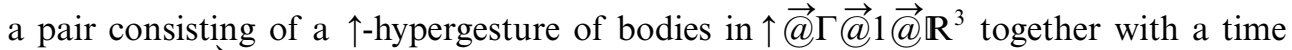
gesture in $\uparrow @ \vec{Q} \mathbb{R}$. Figure 6 shows four stages of a deformation of a body of type $\Sigma \vec{@} 1 \vec{a} \mathbb{R}^{3}$, where $\Sigma$ is a digraph with four vertices $z, w_{1}, w_{2}, w_{3}$ and three arrows $a_{i}: z \rightarrow$ $w_{i}, i=1,2,3$.

In this example, we may reinterpret the hypergesture in $\uparrow \vec{@} \Sigma \vec{@} 1 \vec{@}, \mathbb{R}^{3}=\uparrow \Sigma 1 \vec{@}, \mathbb{R}^{3}$ by the use of Proposition 4.1 and its corollary, Corollary 4.2: while our gesture is a curve of $\Sigma$-shaped hypergestures of loops, we may use the isomorphism $\uparrow \Sigma 1 \vec{@} \mathbb{R}^{3} \leadsto 1 \uparrow \Sigma \vec{@} \mathbb{R}^{3}$ to view the hypergesture in question as being a loop of hypergestures that are curves of $\Sigma$-shaped gestures in $X$.

\section{Natural gestures}

We now proceed to the construction of a gesture for each digraph, and this in a socalled natural way, i.e. such that the category of digraphs is related to the associated gestures in a structurally compatible way, which mathematicians call 'natural' (see [26] for this background). We have spatialization functor Space: Digraph $\rightarrow$ SpaceDigraph as follows: we first take the colimit $|\Delta|$ of the following diagram of topological spaces (see figure 7). For every arrow of $\Delta$, we take one copy of the unit line $I=[0,1]$, and for each vertex one copy of the singleton space $\{*\}$. Then we take the maps from the singleton spaces to the line copies $* \mapsto 1$ or $* \mapsto 0$ for each coincidence of arrow heads or tails and arrows.

The colimit topology is this: the space $|\Delta|$ is all the copies of the unit line $I$ being glued together in their common vertices, and the open sets in $|\Delta|$ are the sets intersecting in an open set for each line $I$. This space is in fact a metric space, i.e. we view it as being given the induced metric from $\mathbb{R}^{V_{\Delta}} \oplus \mathbb{C}^{A_{\Delta}}$ on the subspace consisting of basis vectors $e_{v}$ indexed by vertices $v \in V_{\Delta}$, the arrow $a \in A_{\Delta}$ is realized by a Cartesian product of the line $(1-s) e_{t}+s e_{h}, s \in I$ for an arrow $t \stackrel{a}{\rightarrow} h$ times the unit circle $S_{1}=\exp \{2 \pi i s\}, s \in I$ in $\mathbb{C}$ indexed by the arrows. This means that we have a twisted line $\left([1-s] e_{t}+s e_{h}, \exp \{2 \pi i s\}\right)$, $s \in I$ in the direct sum $c_{a}(t)=\mathbb{R} e_{t} \oplus \mathbb{R} e_{h} \oplus \mathbb{C} e_{a} \subset \mathbb{R}^{V_{\Delta}} \oplus \mathbb{C}^{A_{\Delta}}$. We write $\vec{\Delta}=|\vec{\Delta}|=I @|\Delta|$. The spirals serve two needs: (1) they disambiguate arrows that share heads and tails; and (2) they make loops looking like circles, and consequently spirals for 'non-loops' are the most natural solution to 'draw' them. 

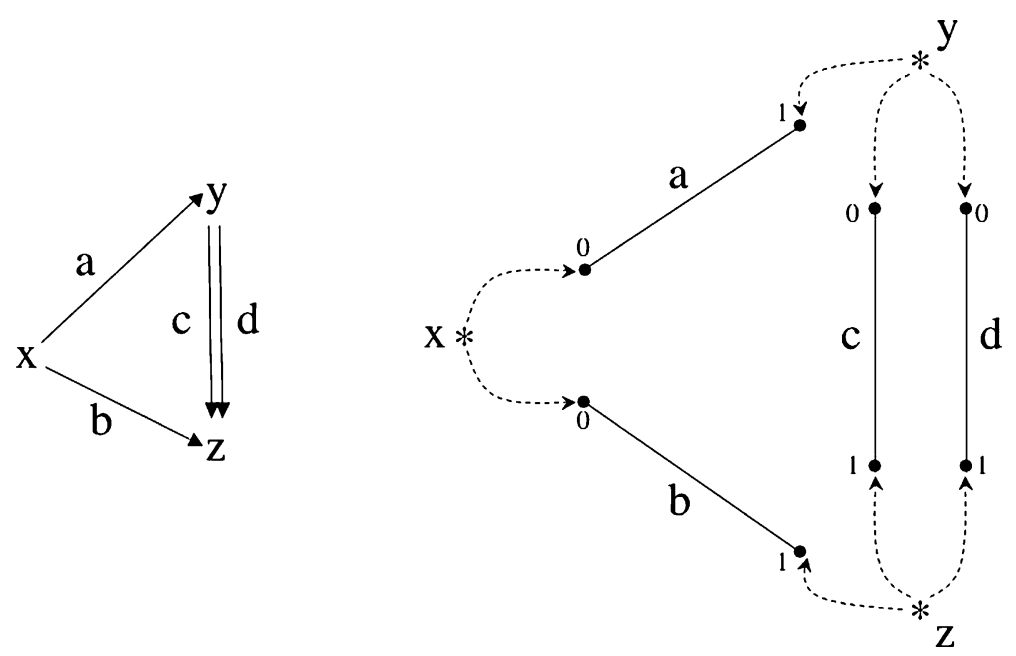

Figure 7. To the right the diagram for the colimit topology derived from the digraph to the left.

Moreover, we have a natural transformation $g: \operatorname{Id}_{\text {Digraph }} \rightarrow \vec{?}$, which means that there is a gesture $g(\Delta): \Delta \rightarrow \vec{\Delta}$ for each digraph, which varies functorially. The gesture maps the vertices $v$ of $\Delta$ to the points $g(\Delta)(v)=e_{v}$ of $|\Delta|$, and the arrows $t \stackrel{a}{\rightarrow} h$ to the curves $g(\Delta)(a)=c_{a}$ in $|\Delta|$ (to ease intuitive understanding, we use the representation in the metric space $\mathbb{R}^{V_{\Delta}} \oplus \mathrm{C}^{A_{\Delta}}$, but in reality we refer to the corresponding structures in the defining colimit). The gesture $g(\Delta): \Delta \rightarrow \vec{\Delta}$ has the following universal property.

Proposition 5.1 For any morphism $f: \Delta \rightarrow \Gamma$ of digraphs and any gesture $\gamma: \Gamma \rightarrow \vec{X}$, there is a unique continuous map $|f|:|\Delta| \rightarrow X$ such that its associated digraph morphism $\vec{f}=|\vec{f}|: \vec{\Delta} \rightarrow \vec{X}$ induces a morphism $g(f): g(\Delta) \rightarrow \gamma$ of gestures.

This follows by standard arguments from the universal property of the colimit topology on $|\Delta|$. In fact, the identity maps on the unit intervals defining the colimit $|\Delta|$ must be mapped into the curves defining the getsure $\gamma$, and this uniquely determines the morphism $|f|$ by the universal property of colimits.

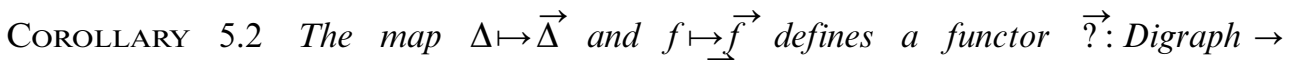
SpaceDigraph and the gesture $g(\Delta): \Delta \rightarrow \vec{\Delta}$ defines a natural transformation $g: I d_{\text {Digraph }} \rightarrow \vec{?}$, also called the natural gesture associated with the digraph $\Delta$.

COROllary 5.3 The gesture $g(\Delta): \Delta \rightarrow \vec{\Delta}$ defines a functor $g:$ Digraph $\rightarrow$ Gesture that is right adjoint to the projection $p:$ Gesture $\rightarrow$ Digraph, in symbols $g \dashv p$. This means that

$$
\operatorname{Digraph}(\Delta, p(\gamma)) \stackrel{\sim}{\rightarrow} \operatorname{Gesture}(g(\Delta), \gamma)
$$

is a bijection, which is functorial in both arguments $\Delta, \gamma$. 


\section{Gestoids}

Given a topological space $X$, consider the category $H_{X}$ of homotopy classes of curves. Its objects are the elements of $X$, while the morphism set $H_{X}(x, y)$ is the set of homotopy classes of curves starting in $x$ and terminating in $y$. The composition of homotopy classes is the homotopy class of the composed curves. Clearly, this is a groupoid, the inverse of a curve class $[\gamma]$ being the class $\left[\gamma^{*}\right]$ of the inverted curve $\gamma^{*}(t)=\gamma(1-t)$. In particular, the group $\mathrm{H}_{x}(x, x)$ is the fundamental group $\pi_{1}(x, X)$ of $X$ in $x$. The category $H_{x}$ is called the fundamental groupoid of $X$. If $\delta: \Delta \rightarrow \vec{X}$ is a gesture, the groupoid generated by the arrows and point of $\delta$ via the canonical morphism $\Delta \stackrel{\delta}{\rightarrow} \vec{X} \rightarrow H_{X}$ is denoted by $H_{\delta}$ and called the fundamental groupoid of $\delta$.

Example 6.1 If $\Delta=1$ is the final digraph, and if $\delta: 1 \rightarrow \vec{X}$ is a loop $\delta(T)$ in $x$, then $H_{\delta}$ is the subgroup of the fundamental group $\pi_{1}(x, X)$ generated by the homotopy class of $\delta(T)$.

We now linearize* the fundamental groupoid over a commutative ring $R$, i.e. the sets $H_{X}(x, y)$ are taken as a basis over $R$, and the composition is defined by bilinear extension from the given basis composition. We call this category $R-H_{X}$ the $(R$-) gestoid of $X$.

The linearized image of the fundamental groupoid $H_{\delta}$ of a gesture $\delta$ is called the $\left(R\right.$-)fundamental gestoid of $\delta$ and denoted by $\mathrm{R} \mathcal{G}_{\delta}$. Intuitively, this is the portion of the gestoid of $X$ that is covered by arrows from the given gesture. So the gestoid is a linearized groupoid.

Example 6.2 In the above Example 6.1, the fundamental gestoid $\mathbb{R} \mathcal{G}_{\delta}$ over the reals is the group algebra $\mathbb{R} H_{\delta}$.

From this construction, the category $R$-Gestoid of $R$-gestoids is the following. The objects, called gestoids, are the $R$-linear categories $G$, i.e. we have bilinear composition, addition, and scalar multiplication of morphisms on the $R$-modules $x @ y$ of morphisms. A morphism $q: G \rightarrow H$ is a linear functor, i.e. all maps $q(x, y): x @ y \rightarrow q(x) @ q(y)$ are $R$-linear. Moreover, every endomorphism $R$-algebra is a group algebra, i.e. there is a

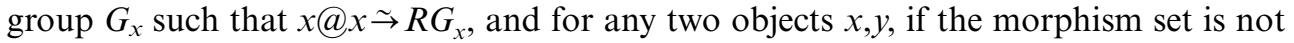

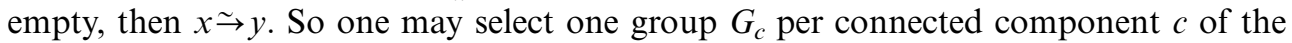
gestoid to describe the group algebras $x @ x \stackrel{\sim}{\rightarrow} R G_{c}$ in that component.

In this way we have associated a gestoid in $R$-Gestoid to a topological space $X$, and then also to a $\Delta$-gesture $\delta: \Delta \rightarrow \vec{X}$ in $X$. Let us check that this assignment completes to functors on the respective categories. To begin with, if $f: X \rightarrow Y$ is continuous, then it maps homotopic curves to homotopic curves, and we deduce a functor $H_{f}: H_{X} \rightarrow H_{Y}$ and then by linear extension a linear functor $R-H_{f}: R-H_{X} \rightarrow R-H_{Y}$, i.e. a morphism of $R$-gestoids.

Finally, if $f: \delta \rightarrow \gamma$ is a morphism of gestures, then the associated morphism of spatial digraphs are not uniquely determined, but the induced functors on the $R$-gestoids of the given gestures are well defined. So we have the required functor

$$
\mathcal{G}: \text { Gesture } \rightarrow \text { Groupoid } \rightarrow R-\text { Gestoid }
$$

\footnotetext{
* Given a category $\mathcal{C}$ and a commutative ring $R$, its linearization $R \mathcal{C}$ is the category whose objects coincide with the objects of $\mathcal{C}$, while for two objects $X$; $Y$, the morphism set $X @_{R \mathcal{C}} Y$ is the free $R$-module generated by the set $X @_{\mathcal{C}} Y$ of -morphisms from $X$ to $Y$, and the composition of morphisms in $R \mathcal{C}$ is the bilinear extension of the composition in $C$.
} 
that connects the topological level of gestures via the level of groupoids from algebraic topology to its linearized version of gestoids.

\subsection{Fundamental groups, networks and Fourier theory}

One interesting aspect in this construction is that the $R$-gestoid of a gesture is based upon groupoids, and these are essentially groups (the automorphism groups in the objects) plus isomorphisms that induce group isomorphisms via conjugation. But this is akin to the background structure of group diagrams used for local networks, and in particular for K-nets. So one might ask whether it is possible to generate gestures that give rise to groupoids relating in a canonical way to given local networks.

This is a central topic in the overall strategy of gestural constructions, since we would like to relate gestures to the abstract algebra of networks or other algebraic concepts, which at first sight have nothing to do with gestures. This concern is intimately related to the fundamental problem of New AI: 'How is it possible to (re)build symbolic thinking from instances of embodiment?' How is it in particular possible to rebuild abstract algebra from gestures? In our context, we have made a step in this direction by use of the powerful tool of fundamental groupoids from algebraic topology. But it is still not a concrete result insofar as the role of this construction is not made explicit or applied to specific problems or constructs from mathematical music theory.

We therefore want to investigate the possible groupoids that intervene in the gestoid construction. Such a reconstruction would entail that, intuitively speaking, we were able to remodel abstract algebraic processes in terms of gestural dynamics. We believe that, in fact, understanding abstract algebra is strongly enhanced (if not enabled) if it uses gestural embodient. This is what Cavaillès [15] seems to suggest (see our citation from our introduction). For music, this would mean that we could envisage the question of how to 'perform' abstract algebraic structures. This is a deep question, since making music is intimately related to the expression of thoughts. So we would like to be able to express algebraic insights, revealed by use of K-nets or symmetry groups, for example, in terms of musical gestures. To put it more strikingly: 'Is it possible to play the music of thoughts?'

Now, every finitely generated abelian group is the fundamental group of a topological space. This follows from the fact that such a group is a finite product of cyclic groups, that the fundamental group of a product of topological spaces is the product of the fundamental groups of the factors, and that a finite cyclic group $\mathbb{Z}_{n}$ is the fundamental group of the lens space $L_{n, 1}$, which is the quotient $S^{3} / \mathbb{Z}_{n}$ of the 3 -sphere $S^{3} \subset \mathbb{C}^{2}$ modulo the group action $k \cdot(z, w)=\left(u^{k} z, u^{k} w\right), u=\mathrm{e}^{2 \pi i / n}$ [27, Example 7.15], whereas $\mathbb{Z}$ is the fundamental group of the circle $S^{1}$ (or of $\mathrm{SO}(2)$, etc.). Therefore, in particular, the classical pitch class group $\mathbb{Z}_{12}$ is in fact a fundamental group, namely that of the Cartesian product $L_{3,1} \times L_{4,1}$. Musically speaking this implies the following fact.

Fact 6.3 All the finitely generated abelian groups, in particular the pitch class groups $\mathbb{Z}_{12}$, defining $\mathrm{K}$-networks, may be realized via fundamental groups at the level of topological spaces.

This is a possibility that is most relevant for the problem of effectively playing such networks. But let us explain the situation. We have just learnt that important abstract groups are realized as fundamental groups of topological spaces. This means that curves in topological spaces may represent elements of such abstract groups, whereas 
the concatenation of such curves realizes abstract group operations. Since the generators of our groups are finite in number, this opens the question of whether we may find gestures such that their arrows are associated with the generators of our groups. And whether it is possible to give an interpretation of the involved topological spaces - in particular, lens spaces - in terms of spaces of more or less complex bodily gestures. This is an open question. But lens spaces are objects of low dimension and may probably intervene for gestures of conductors' or musicians' limbs. This is plausible from our previous work on the pianist's hand as described in Example 3.2.

For music theory, the best situation would be the following.

CONJECTURE 6.4 For every K-net, there is a gestoid of the same digraph that contains a $K$-net isomorphic to the given K-net.

Let us make this conjecture more precise to give a suggestion of how a K-net could be interpreted within a gestoid. To this end, recall that an $\mathbb{R}$-gestoid has a group algebra $x @ x=\mathbb{R} \pi_{1}(x, X)$ for each of its objects $x$, and that for any path $f: x \rightarrow y$ we have a conjugation isomorphism of $\mathbb{R}$-algebras Int $: x @ x \sim y @ y: z \mapsto f \circ z \circ f^{-1}$. So we are in the category $A \lg _{\mathbb{R}}$ of $\mathbb{R}$-algebras, and the given gesture $\gamma: \Gamma \rightarrow \vec{X}$ defines a diagram $D_{\gamma}$ of $\mathbb{R}$-algebras, the algebra $\mathbb{R} \pi_{1}(x, X)$ at point $x$, and algebra isomorphisms. To give a K-net means to select an element of $\lim D_{\gamma}$. In terms of the category $A \lg _{\mathrm{R}}$, this means evaluating the diagram of representable functors $@ \mathbb{R} \pi_{1}(x, X)$ at the address $\mathbb{R} \mathbb{Z}$, since $\mathbb{R} \mathbb{Z} @ B \sim B^{*}$, where $B^{*}$ is the group of multiplicatively invertible elements of $B$. If we have a space $X$ with $\pi_{1}(x, X) \sim \mathrm{Z}_{12}$, then selecting an element $z_{x} \in \mathbb{Z}_{12}$ corresponds to a point in $\mathbb{R} \mathbb{Z} @ B \sim \stackrel{\sim}{\rightarrow} B^{*}$ for $B=\mathbb{R} \mathbb{Z}_{12}$. The combination of an algebra isomorphism Int $_{f}$ with a multiplication by a group element simulates the affine morphisms used in K-net theory. Therefore this structure yields a model for K-nets in finitely generated abelian groups.

The intriguing point in this presentation is that the musical interpretation of elements $z \in \mathbb{Z}_{12}$ as pitch classes seems to be somewhat mysterious when reinterpreting $\mathbb{Z}_{12} \stackrel{\sim}{\rightarrow} \pi_{1}(x, X)$. Why should a multiple loop be associated with a pitch?

There is however a very natural interpretation of this mystery in terms of Fourier theory. If a periodic time function $x(t)$ with frequency 1 is represented by a Fourier series $x(t)=\Sigma_{n} \gamma_{n} \mathrm{e}^{\mathrm{i} 2 n t}$ with finitely many non-vanishing coefficients $\gamma_{n}$, the functions $\varepsilon_{n}(t)=\mathrm{e}^{\mathrm{i} 2 \pi n \mathrm{t}}$ are linearly independent, and $\varepsilon_{n}=\varepsilon_{1}^{n}$ for $n \in \mathbb{Z}$. Therefore the function $x$ is in fact an element of the group algebra $\mathbb{C} \mathbb{Z}$, if we identify $\varepsilon_{n}$ with $n \in \mathbb{Z}$. It is now easy to reinterpret the elements of $\mathbb{Z}$ in a natural way as being elements of a fundamental group.

In fact, consider the circle $S^{1}$, which we identify with the unitary group $U \subset \mathbb{C}$ of complex numbers of length one. Then a gesture $\delta: 1 \rightarrow \vec{S}^{1}$ is a loop in $S^{1}$. In particular, we have the gesture $\varepsilon: 1 \rightarrow \varepsilon_{1}$ associated with the loop $\varepsilon_{1}: I \rightarrow S^{1}$ in $1 \in U$. This yields the fundamental component of the Fourier representation, and it is a generator of the fundamental group $\pi_{1}\left(1, S^{1}\right) \stackrel{\sim}{\rightarrow}$. So the Fourier representation corresponds to the formal sum $\Sigma_{n} \gamma_{n} \varepsilon^{n}$ in the complex gestoid $\mathbb{C G}_{\varepsilon}$, i.e. the linearization of the fundamental groupoid over the complex numbers. In short, we have the following fact.

Fact 6.5 The Fourier representation is the linear combination in the complex gestoid $\mathbb{C G}_{\varepsilon}$ of gesture classes that are powers of the fundamental loop gesture in 1.

The converse, i.e. the reinterpretation of elements in more general gestoids in terms of time functions, is a challenging problem. In particular, we are asked to reinterpret the 
loops in the fundamental group $\mathbb{Z}_{m}$ of a lens space $L_{m, 1}$ in terms of acoustically meaningful functions.

\section{Spectroids and natural formulae}

From the category Digraph of digraphs, one may also derive algebraic instead of topological structures. On one side, this is motivated by the well-known dichotomy of topology versus algebra as guiding paradigms in mathematics. On the other side, as we shall see in Section 7.1, an algebraic perspective on digraphs is musically motivated by the theory of local musical networks.

Our approach refers to Gabriel's construction of spectroids* from digraphs [14,28], which runs as follows. Given a commutative artinian ring $k$ the category $k$-Spectroid of $k$-spectroids has the $k$-spectroids as objects: they are $k$-linear categories $S$, where any two different objects $x \neq y$ are non-isomorphic, where the endomorphism algebras $x @ x$ of all objects $x$ are local -i.e. the non-invertible endomorphisms of $x$ are a (two-sided) ideal Rad $(x) \subset x @ x$ - and such that the morphism spaces $x @ y$ have finite length over $k$. We shall henceforth omit the finiteness condition except when explicitely stated. The morphisms between $k$-spectroids are the local $k$-linear functors $f: S \rightarrow T$, i.e. those carrying the radical $\operatorname{Rad}(S)$ (by definition the non-invertible morphisms of $S$ ) into $\operatorname{Rad}(T)$. In the what follows, we fix $k=\mathbb{R}$ as the base ring of spectroids and omit this specification.

The analogous construction for gestures is an algebraic interpretation of digraphs instead of a topological one. We interpret vertices as objects and arrows as morphisms in spectroids. More precisely, a digraph is called radical iff it is the digraph $\operatorname{Rad}(S)$ of a spectroid $S$ having the noninvertible morphisms as its arrows and the domain and codomain maps $d, c: \operatorname{Rad}(\mathrm{s}) \Rightarrow S$ as tails $d=t$ and heads $c=h$ of arrows. The category RadicalDiagraph of radical digraphs has the radical digraphs $\operatorname{Rad}(S)$ as its objects and the graph morphisms $r: \operatorname{Rad}(S) \rightarrow \operatorname{Rad}(T)$ induced by morphisms $s: S \rightarrow T$ of the underlying spectroids, we then write $r=\operatorname{Rad}(S)$. This category is the algebraic analogue of the category SpaceDigraph of spatial digraphs. A morphism of digraphs $\phi: \Delta \rightarrow$ $\operatorname{Rad}(S)$ is called a $\Delta$-formula in $S$.

Example 7.1 Given a digraph $\Delta$, we have the $\mathbb{R}$-category $\mathbb{R} \Delta$, which is the linearized path category ${ }^{\dagger} \mathbb{R} \Delta=\mathbb{R} \operatorname{Path}(\Delta)$. Consider the digraph

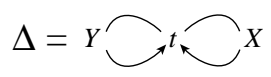

and the quotient category $S$ obtained from $\mathbb{R} \Delta$ by division through the ideal generated by the relations $X^{2}-Y X, Y^{2}-X Y, X Y+Y X, X^{3}$. This means that we have a threedimensional radical $\operatorname{Rad}(S)=\mathbb{R} x+\mathbb{R} y+\mathbb{R} x y$, with the relations $x^{2}=y x=-y^{2}, x y=$ $-y x$, and $x^{3}=0$. Now, if we are given a digraph $\Gamma$, a formula $\psi: \Gamma \rightarrow \operatorname{Rad}(S)$ is the

\footnotetext{
* The term spectroid is akin to spectrum. This is intentional since, by Gabriel's thesis [14], one has the following fact: for $B$ a $k$-algebra over an artinian commutative ring $k$, consider the category $\operatorname{Mod}_{B}$ of right $B$-modules of finite $k$-length, and take the full subcategory $S p\left(\operatorname{Mod}_{B}\right)$ of representatives of all indecomposable injective modules. Then if every right ideal of $B$ is two-sided, there is a bijection between the objects of $\operatorname{Sp}\left(\operatorname{Mod}_{B}\right)$ and the prime spectrum $\operatorname{Spec}(B)$, i.e. the set of two-sided prime ideals of $B$ (meaning that any inclusion $a I b \subset I$ for such an ideal $I$, and $a, b \in B$, implies $a \in I$ or $b \in I$ ).

$\dagger$ The path category of a digraph $\Delta$ has the vertex set $V_{\Delta}$ as the object set and the paths $x \rightarrow x^{\prime} \rightarrow \ldots y$ as morphisms from vertex $x$ to vertex $y$. The composition of morphisms is the composition of paths, while the lazy path of length 0 in $x$ is the identity in $x$.
} 
assignment of any vector $\psi(a) \in \operatorname{Rad}(S)$ to an arrow $a \in A_{\Gamma}$, since there is only one vertex in $\operatorname{Rad}(S)$. Then the formula is completely described by labeling the arrows of $\Gamma$ with their images and writing down the relations in $S$. For example, if $\Gamma=1$, and if the $\psi$-image of $T$ is $\psi(T)=x y+3 y^{2}$, then we may write the formula as

$$
\psi=x y+\overbrace{}^{3 y^{2}} t, \quad\left(x y+3 y^{2}\right)^{2}=0 .
$$

Given two formulae $\phi: \Delta \rightarrow \operatorname{Rad}(S), \psi: \Gamma \rightarrow \operatorname{Rad}(T)$ a morphism $f: \phi \rightarrow \psi$ of formulae is a morphism $f: \Delta \rightarrow \Gamma$ of digraphs such that there is a morphism $\operatorname{Rad}(S): \operatorname{Rad}(S) \rightarrow$ $\operatorname{Rad}(T)$ of radical digraphs that commutes with $f$, i.e. $\psi \circ f=\operatorname{Rad}(s) \circ \phi$. The category of formulae is denoted by Formula, and we have a canonical projection $q$ : Formula $\rightarrow$ Digraph by forgetting about the radical codomain.

For a digraph morphism $f: \Delta \rightarrow \Gamma$, we have the associated functor $\operatorname{Path}(f): \operatorname{Path}(\Delta) \rightarrow$ $\operatorname{Path}(\Gamma)$ and its linearized extension $\mathbb{R} f: \mathbb{R} \Delta \rightarrow \mathbb{R} \Gamma$. This defines a functor $\mathbb{R}$ ? : Diagraph $\rightarrow$ Spectroid, and then, by restriction to the radicals, a functor Rad: Digraph $\rightarrow$ RadicalDigraph $: \Delta \mapsto \operatorname{Rad}(\Delta)=\operatorname{Rad}(\mathbb{R} \Delta)$. For every digraph $\Delta$, we have a canonical formula $r(\Delta): \Delta \rightarrow \operatorname{Rad}(\Delta)$ given by the identity on the arrows and vertices.

Proposition 7.2 For any morphism $f: \Delta \rightarrow \Gamma$ of digraphs and any formula $\psi: \Gamma \rightarrow$ $\operatorname{Rad}(S)$, there is a unique functor $\mathbb{R} f: \mathbb{R} \Delta \rightarrow S$ such that its associated digraph morphism $\operatorname{Rad}(f): \operatorname{Rad}(\Delta) \rightarrow \operatorname{Rad}(S)$ induces a morphism $r(f): r(\Delta) \rightarrow \psi$ of formulae.

COROLlary 7.3 The map $\Delta \mapsto \operatorname{Rad}(\Delta)$ and $f \mapsto \operatorname{Rad}(f)$ defines a functor Rad : Digraph $\rightarrow$ RadicalDigraph and the formula $r(\Delta): \Delta \rightarrow \operatorname{Rad}(\Delta)$ defines a natural transformation $r: I d_{\text {Digraph }} \rightarrow$ Rad, also called the natural formula associated with the digraph $\Delta$.

COROLlaRy 7.4 The formula $r(\Delta): \Delta \rightarrow \operatorname{Rad}(\Delta)$ defines a functor $r:$ Digraph $\rightarrow$ Formula which is right adjoint to the projection $q:$ Formula $\rightarrow$ Digraph, in symbols $r \dashv q$. This means that

$$
\operatorname{Digraph}(\Delta, q(\psi)) \stackrel{\sim}{\rightarrow} \operatorname{Formula}(r(\Delta), \psi))
$$

is a bijection, which is functorial in both arguments $\Delta, \psi$.

\subsection{Local networks are solutions of representations of natural formulae}

If we are given a formula, it is natural to ask for its solutions. We do not elaborate this subject here, but since it is an essential technique for the construction of (local) networks, ${ }^{*}$ it is necessary to indicate the relation to those concepts that are important in music theory [4,29]. A spectroid is deduced from the situation of a category $\mathbf{M o d}_{k}$ of $k$-modules over a commutative ring $k$. Then the morphism sets $M @ N$ are $k$-modules and the composition is bilinear. One selects a complete set of representatives of isomorphism classes of indecomposable injective modules and considers the full subcategory $S$ defined by these objects. Therefore a formula $\psi: \Gamma \rightarrow \operatorname{Rad}(S)$ defines a diagram of morphisms in $S$, which is a special case of a diagram $\psi: \Gamma \rightarrow \mathbf{M o d}_{k}$ as considered in network theory. We therefore call $\psi$ a generalized formula. Now, this is

\footnotetext{
* Recall that a local network is described by a diagram of modules $M_{i}$ and affine module homomorphisms, together with an element $m_{i} \in M_{i}$ for each vertex module $M_{i}$, such that any two such elements are mapped to each other by the given homomorphisms. In other words, a local network is an element of the limit of the given diagram. See [4] for more details on this construction.
} 
equivalent to giving a $k$-linear functor $k \psi: k \Gamma \rightarrow \operatorname{Mod}_{k}$ defined on the spectroid $k \Gamma$. But if we take the natural formula $r(\Gamma): \Gamma \rightarrow \operatorname{Rad}(\Gamma), k \psi$ defines an obvious morphism $f(\psi)$ : $f(\Gamma) \rightarrow \psi$. And if we are given relations $R$ among the arrows associated with $\psi$, this means that we may factorize $f(\psi)$ through the quotient category $k \Gamma / R$ and instead give a morphism $f(\psi) / R: r(\Gamma) / R \rightarrow \psi$. Therefore, the generalized formulae in $\mathbf{M o d}_{k}$ are 'representations' of natural formulae or quotients thereof.

To deal with solutions, we need points in the modules that are the vertex images of the generalized formula. To this end, one selects a module $A$ (an address module in the theory of denotators [1]) and then evaluates the representable functors @ $\psi(x)$ of the vertex modules $\psi(x)$ at $A$, whence a diagram $A @ \psi$ of sets $A @ \psi(x)$, which are related by the evaluated morphisms $A @ \psi(a)$ for arrow $a$ of $\Gamma$. A solution of the generalized formula $\psi$ at address $A$ is, by definition, an element $s$ of the limit A@lim $\psi$ of this set diagram. This is precisely what we called a local network in the theory of networks [4]. Summarizing, we have the following fact.

Fact 7.5 Local networks are the solutions of generalized formulae at selected addresses $\mathrm{A}$.

\section{Tangent categories}

In algebraic geometry, the Zariski tangent space $T_{X, x}$ of an $\mathbb{R}$-rational point of an $\mathbb{R}$-scheme is the $\mathbb{R}$-linear dual $\left(\mathrm{m} / \mathrm{m}^{2}\right)^{*}$ of the quotient $\mathrm{m} / \mathrm{m}^{2}$ of the maximal ideal $\mathrm{m}$ of the local ring $\mathcal{O}_{X, x}$. In an $\mathbb{R}$-spectroid $S$, every endomorphism algebra $x @ x$ is local with maximal ideal $m_{x}=\operatorname{Rad}(x)$. So we may associate the following linear category with tangent spaces: consider the quotient category $S / \operatorname{Rad}(S)^{2}$ and take its linear subcategory $T_{S}$, which for $x \neq y$ has $T_{S}(x, y)=\operatorname{Rad}(S)(x, y) / \operatorname{Rad}(S)^{2}(x, y)$, while $T_{S}(x, x)=\mathbb{R} \oplus \operatorname{Rad}(x) / \operatorname{Rad}^{2}(x)$. So the endomorphisms in $T_{S}(x, x)$ are identified with the formal sums $\mu+\varepsilon . t, \mu \in \mathbb{R}, t \in \operatorname{Rad}(x) / \operatorname{Rad}^{2}(x)$, which are added component-wise and multiplied under the infinitesimal condition $\varepsilon^{2}=0$ via $(\mu+\varepsilon . t) \circ(v+\varepsilon . s)=\mu v+$ $\varepsilon$. $(v t+\mu s)$. If, for $x \neq y, f \in T_{s}(x, y)$, then we formally write $\varepsilon . f$ instead of $f$ and then $\varepsilon . f \circ(\mu+\varepsilon . t)=\varepsilon . \mu f$. This means that we view the tangent category as built from 'tangent vectors', as indicated by the $\varepsilon$-coefficient.

If $f: S \rightarrow U$ is a morphism of spectroids, then, being local, it factorizes to $f_{R}: S /$ $\operatorname{Rad}^{2}(S) \rightarrow U / \operatorname{Rad}^{2}(U)$ and then evidently also takes the tangent subcategories into one another, yielding a functor $T_{f}: T_{S} \rightarrow T_{U}$. This defines a functor on the category of spectroids.

Example 8.1 If $S=\mathbb{R} 1$ with the final digraph $1=t \quad T$, then $T_{S}$ looks as follows: it has just one object $x$ and its endomorphisms $T_{S}(x, x)$ are the algebra $\mathbb{R}[\varepsilon]$ of dual numbers $\mu+\varepsilon . t, \mu, t \in \mathbb{R}$. Such a number represents the tangent of length $t$ at the point $\mu$ of the affine line $\mathbb{A}$ over the reals.

Then, if we are given a formula $\psi: \Gamma \rightarrow \operatorname{Rad}(S)$, we consider the morphisms in $T_{S}$ defined by $\psi$ as follows: for $x \neq y$ and an arrow $x \stackrel{a}{\rightarrow} y$, we take $\psi^{a}=\varepsilon$. $\left(\psi(a)\right.$ mod) $\operatorname{Rad}^{2}$, and for a loop $x \stackrel{a}{\rightarrow} x$, we take $\psi^{a}=1+\varepsilon .\left(\psi(a) \bmod R a d^{2}\right)$. Therefore, in the tangent category $T_{S}$, we have the exponential rule for endomorphisms on $x$, i.e. for two loops $x \stackrel{a, b}{\rightarrow} x$, we have $\psi^{a+b}=\psi^{a} \circ \psi^{\mathrm{b}}$, while for $x \neq y$, loops $x \stackrel{a}{\rightarrow} x, y \stackrel{c}{\rightarrow} y$, and an arrow $x \stackrel{b}{\rightarrow} y$, we have $\psi^{b} \circ \psi^{a}=\psi^{b}=\psi^{c} \circ \psi^{b}$. Denote then by $T_{\psi}$ the subcategory of $T_{S}$ generated by the arrows $\psi^{a}, a \in A_{\Gamma}$ and their inverses-if they exist-and call this the tangent category of the formula $\psi$. The inverses are of course precisely the automorphisms 
$\left(\psi^{a}\right)^{-1}=1+\varepsilon .\left(-\psi(a) \bmod \operatorname{Rad}^{2}\right)$, which we may also think of as being generated by new 'inverse arrows' $-a$ on $\Gamma$, much as it is done in the Bass-Serre theory of graphs of groups [30]. So we also write $\psi^{-a}=1+\varepsilon .\left(-\psi(a) \bmod R a d^{2}\right)$ for these inverses. Observe that for a formula $\psi: \Gamma \rightarrow \operatorname{Rad}(S)$ of a finite digraph $\Gamma$, the tangent category $T_{\psi}$ has only finitely many morphisms between different objects and the endomorphism monoids are finitely generated commutative groups.

By analogy with the fundamental group construction on the topological branch of our discussion, we may now linearize the tangent category $T_{\psi}$ of a formula. We denote this by $\mathcal{F}_{\psi}$ and call it the radical formoid of the formula $\psi$. In the above-mentioned situation of a finite digraph of the formula $\psi$, the radical formoid has finitedimensional morphism vector spaces $x @ y$ for different objects $x, y$, whereas the endomorphism spaces are group algebras $x @ x \sim \mathbb{R} T_{\psi}(x, x)$ over the finitely generated commutative groups $T_{\psi}(x, x)$.

Given two formulae $\psi: \Gamma \rightarrow \operatorname{Rad}(S), \phi: \Delta \rightarrow \operatorname{Rad}(U)$ and a morphism $f: \psi \rightarrow \phi$ of formulae, we have an auxiliary morphism $s: S \rightarrow U$ of spectroids such that $\operatorname{Rad}(s)$ commutes with $f: \Gamma \rightarrow \Delta$. So for any arrow $a \in A_{\Gamma}$, we have $s(\psi(a))=\phi(f(a))$. Moreover, $s\left(1_{x}\right)=1_{s(x)}$. Therefore for the residual categories modulo $\operatorname{Rad}^{2}$, we have the residual functor $s / \operatorname{Rad}^{2}: S / \operatorname{Rad}(S)^{2} \rightarrow U / \operatorname{Rad}(U)^{2}$, which on loops $x \rightarrow x$ in $x$ acts by $s / \operatorname{Rad}^{2}\left(1_{x}+\right.$ $\left.\psi(a) \bmod R a d^{2}\right)=1_{s(x)}+s(\psi(a)) \bmod \operatorname{Rad}^{2}=1_{s(x)}+s\left(\phi(f(a)) \bmod \operatorname{Rad}^{2}\right.$, and on arrows $x \stackrel{a}{\rightarrow} y$ for $x \neq y$ yields $s / \operatorname{Rad}^{2}\left(\psi(a) \bmod \operatorname{Rad}^{2}\right)=\phi(f(a)) \bmod \operatorname{Rad}^{2}$. This shows that we have a functor $\mathcal{F}_{f}: \mathcal{F}_{\psi} \rightarrow \mathcal{F}_{\phi}$ associated with $f$, and this assignment is obviously functorial. Therefore we have a functor $\mathcal{F}:$ Formula $\rightarrow \mathbb{R}-$ Formoid, the latter category being, by definition, the category of linear categories generated from subcategories of residual categories mod $\mathrm{Rad}^{2}$ of spectroids.

Example 8.2 In the above Example 8.1, if we take the natural formula $r(1): 1 \rightarrow$

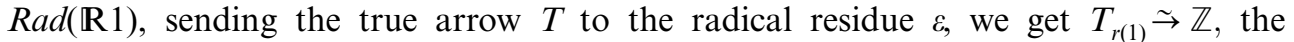
integer $z$ corresponding to the power $1+\varepsilon . z$ in $\mathbb{R}[\varepsilon]$.

We are now ready to observe the overall path from the final digraph $1=t T$, when the gestural and formulaic functors are applied. On the one hand, the topological construction of the fundamental gestoid $\mathcal{G}_{g(1)}$ is the category $\mathbb{R} \mathbb{Z}$, the group algebra over $\mathbb{R}$ of the group of integers, and the latter comes in via the generator of the fundamental group of the circle $S^{1}$. On the other hand, the radical formoid $\mathcal{F}_{r(1)}$ is also the group algebra $\mathbb{R} \mathbb{Z}$, where $\mathbb{Z}$ now comes in as the group generated by the 'tangent vector' $1+\varepsilon$, corresponding to the residue $\varepsilon$ of the generating loop $T$ of the linearized path algebra $\mathbb{R} 1 \leadsto \mathbb{R}[T]$ associated with the final digraph 1 . So both constructions yield isomorphic categories

$$
\mathcal{G}_{g(1)} \stackrel{\sim}{\rightarrow} \mathcal{F}_{r(1)}
$$

when taking the gestural or formulaic branch. Of course, this identification of the results from the two paths is not possible for general digraphs, since the gestoid is built from a groupoid, whereas the formoid stems from a spectroid, where different objects are never isomorphic. So for general digraphs $\Gamma$ we have $\mathcal{G}_{g(\Gamma)} \tilde{\mathcal{T}}_{\mathcal{F}_{r(\Gamma)}}$.

This can be made more precise on the endomorphism algebras. The fundamental gestoid $\mathcal{G}_{g(\Gamma)}$ of a digraph $\Gamma$ is easily calculated: it is well known [31 Theorem10.7] that the fundamental group of a graph is free, the number of generators being given by the number of edges added to a spanning tree. So, normally, this is a non-abelian group, whereas the formoid $\mathcal{F}_{r(\Gamma)}$ has abelian groups defining the endomorphism algebras. 


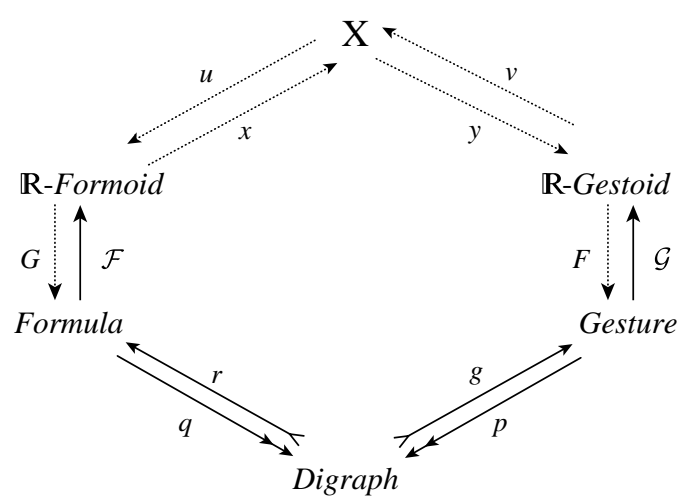

Figure 8. Diamond diagram.

\section{The diamond conjecture}

The preceding constructions are by no means in a complete theoretical shape. For example, the formulaic branch does not rely on the real numbers - any commutative field $k$ or artinian commutative ring would equally provide a reasonable setup. For example, the formoids over the fields of positive characteristic $p$ yield group algebras $\mathcal{F}_{r(1)} \stackrel{\sim}{\rightarrow} k Z_{p}$ over the cyclic group $\mathbb{Z}_{p}$, and this algebra would be needed to reflect the appearance of fundamental groups $\mathbb{Z}_{p}$ for lens spaces, as mentioned above. Moreover, the reduction modulo $\mathrm{Rad}^{2}$ is not mandatory, more general reductions modulo $\mathrm{Rad}^{2}$, $n>2$ would also yield interesting information. For example, we could take care of paths in categories $k \Gamma$ longer than just those of length one.

In order to shape the hypothetical ideal architecture, which should encompass and harmonize the two branches, we present the following diamond diagram. It is topped by a hypothetical category $X$, which should unite the algebraic and the topological branches. The hypothetical functors $G, F, u, v, x, y$ are shown by dotted arrows. It is further conjectured that, for the functors $G$ and $F$, we have the adjunctions $\mathcal{F} \dashv \mathcal{G}$ and $\mathcal{G} \dashv \mathcal{F}$. Finally, it is conjectured that the ascending/descending arrows can be completed to a commutative diagram by two ascending/descending arrows $x, y / u, v$, as shown in figure 8 .

In other words, starting from a common basis of elementary mathematical structures, i.e. the digraphs, we have a double unfolding of musically relevant structures: gestures and formulae. Our hypothesis is that there exists a 'universe' $X$, whose ontology englobes these two branches in a natural way and at the same time, expresses a unified comprehension of music.

\section{The software agenda: implementation on RUBATO $^{\circledR}$}

The RUBATO ${ }^{\circledR}$ environment (the open-source Java-based software is reachable via [32]) for music analysis, composition and performance has been implemented such that the category Mod $^{@}$ is reasonably manageable, which means that a number of common rings and module constructions, as well as module homomorphisms, can be defined and manipulated, and also presheaves, diagrams of presheaves and limits or colimits over these algebraic objects. 'Reasonably' means that the construction of denotators 
that solve limit constraints are still not available in full generality. This is however not a serious drawback, since the solutions of limits amount to the construction of families of objects that are subject to affine constraints, a topic that pertains to computer algebra and can be implemented on specific rubettes of the software at any time.

This is quite satisfying for the network part of the preceding theory, but for the topological part, where the category Top of topological spaces and continuous maps is used, RUBATO ${ }^{\circledR}$ is not yet up to date. The point is that the denotator formalism is split into two architectural ingredients: the mathematical basis topos, which is used as a starting level in the recursive construction of denotators, and the conceptual framework, which enables the extension of denotators of a given form (type) to new denotator forms. The latter is generic, and its class hierarchy can be reused. But the mathematical basis, i.e. the topos Top ${ }^{\circledR}$, needs to be implemented from scratch as a topic in computer topology.

Besides the purely mathematical targets, this topic includes also writing rubettes for visualization of curves, of gestures, hypergestures and also rubettes for gestural manipulations and interaction, in particular for interaction with instrumental interfaces, be it software instruments or hardware components such as joysticks or tracking devices.

A particular interest lies in routines for the calculation of gestoids, i.e. essentially fundamental groupoids of graphs. The latter is in fact a well-known methodology, since fundamental groups of graphs are a solved combinatorial procedure; see [31].

\section{Conclusions and future research}

This paper demonstrates an interplay of music and mathematics that strongly differs from the usual scheme of reducing mathematics to a toolbox of formal models for music. We have in fact presented an overall framework for the mathematical theory of music, which is ramified into an algebraic and a topological branch, both being rooted in the topos of directed graphs. Whereas the algebraic ramification comprises the universe of formulae, transformations and functional constraints as described by functorial diagrammatic limits, the topological component comprises the continuous aspects of the creative dynamics of musical gestures and their multilayered articulation.

From a mathematical point of view, these two branches unfold in a surprisingly parallel manner, although the concrete structures are fairly heterogeneous. However, the unity of the underlying musical substance suggests that these two apparently divergent strategies should find a common point of unification, an idea that we have described in terms of a conjectural diamond of categories. To support this conjecture, we have also given a number of points of unification. In particular, the passage from the topological branch to the algebraic one is suggested by the idea of the gestoid, which is in fact an 'algebraic' category associated with the fundamental groupoid of a gesture.

The approach described in this paper could be taken as a starting point for the investigation of more general relations between fundamental gestoids and transformational music theory, as suggested by our Conjecture 6.4. Starting from the realization of an abstract group as a fundamental group, conclusions may be derived concerning the realization of the group's elements by musical gestures. This was suggested in our example dealing with the interpretation of the loop digraph gestoid with regard to Fourier analysis. 
Evidently, the algebraic branch of this approach evokes the application of the representation theory of quivers (synonym for digraphs in that context) and corresponding classification theorems, tame and wild quivers, etc.; see also [33] and the classical literature [28].

A final word on the idea of introducing gestural logic as an approach for overcoming the dramatic gap between plain bodily gesticulation and abstract intelligence. We observe that Digraph is a topos, so every digraph $\Delta$ has a canonical logic, i.e. a Heyting algebra on the sub-digraph sets $\operatorname{Sub}(\Delta)=\Delta @ \Omega$, for the subobject classifier $\Omega$. The logical operations on $\operatorname{Sub}(\Delta)$ are as follows. If $\Gamma, \Sigma$ are two sub-digraphs of $\Delta$, then we have $\Gamma \wedge \Sigma=\Gamma \cap \Sigma$ and $\Gamma \vee \Sigma=\Gamma \cup \Sigma$. The implication $\Gamma \Rightarrow \Sigma$ is a bit more involved: we have $V_{\Gamma \Rightarrow \Sigma}=\left(V_{\Delta}-V_{\Gamma}\right) \cup V_{\Sigma}$. The arrows are these: they include $\Gamma \cap \Sigma$, then all arrows on vertices of the intersection $V_{\Gamma} \cap V_{\Sigma}$ that are not in $\Gamma$, further all $\Delta$-arrows on vertices in $V_{\Delta}-V_{\Gamma}$, finally all $\Delta$-arrows between vertices in $V_{\Gamma} \cap V_{\Sigma}$ and vertices in $V_{\Delta}-V_{\Gamma}$.

This Heyting logic is identified with the contravariant functor@ $\Omega \Omega$ : Digraph $\rightarrow$ Heyting with values in the category Heyting of Heyting algebras. The functor also applies to gestures and formulae by applying the logical operations to the respective domains. More precisely, if $\gamma: \Gamma \rightarrow \vec{X}$ is a gesture, then the set of subgestures $\operatorname{Sub}(\gamma)$ of $\gamma$ inherits the Heyting-structure on its domain $\Gamma$. Mutatis mutandis, the analogous Heyting-structure is realized for the set $\operatorname{Sub}(\psi)$ of a formula $\psi$. See [34] for an example of this logic in musical harmony.

This formalism may be used as a logical device for shedding light on the fundamental problem of the New Artificial Intelligence of embodiment.

\section{Acknowledgements}

We would like to thank Yves André and the anonymous reviewers for their accurate reading of the manuscript and their precious suggestions concerning a better presentation of the topic.

\section{References}

[1] Mazzola, G. (Ed.), 2002, The Topos of Music-Geometric Logic of Concepts, Theory, and Performance (Basel: Birkhäuser).

[2] Kan, D., 1958, Adjoint functors. Transactions of the American Mathematical Society, 87, 294-329.

[3] Drossos, C.A., 2005, Sets, categories and structuralism. In G. Sica (Ed.), What is Category Theory? (Monza: Polimetrica).

[4] Mazzola, G. and Andreatta, M., Summer 2006, From a categorical point of view: K-nets as limit denotators. Perspectives of New Music, 44.

[5] Mac Lane, S., 1989, The development of mathematical ideas by collision: the case of categories and topos theory. In: Categorical Topology and its Relation to Analysis, Algebra and Combinatorics (Teaneck, NJ: World Scientific), $1-9$.

[6] Alunni, C., 2005, Diagrammes \& catégories comme prolégomènes à la question: qu'est-ce que s'orienter diagrammatiquement dans la pensée? In: N. Batt (Ed.), Penser par le Diagramme (Paris: Presses Universitaires de Vincennes).

[7] Lewin, D., 1987, Generalized Musical Intervals and Transformations (New Haven, CT: Yale University Press).

[8] Noll, T., 1995, Morphologische Grundlagen der abendländischen Harmonik. Doctoral thesis, Technische Universität Berlin.

[9] Piaget, J., 1990, Morphismes et Categories. Comparer et Transformer (Neuchâtel: Delachaux et Niestlé).

[10] Mac Lane, S., 1986, Mathematics, Form and Function (New York: Springer-Verlag).

[11] Andreatta, M., 2003, Méthodes algébriques en musique et musicologie du XXème siècle: aspects théoriques, analytiques et compositionnels. PhD thesis, Ircam/EHESS.

[12] Landry, E. and Marquis, J.-P., 2005, Categories in context: Historical, foundational, and philosophical. Philosophia Mathematica, 13, 1-43.

[13] Corry, L., 2004, Modern Algebra and the Rise of Mathematical Structures (2nd edn) (Birkhäuser Verlag).

[14] Gabriel, P., 1962, Des catégorie abéliennes. Bulletin de la Société Mathématique de France, 90. 
[15] Batt, N. (Ed.), 2005, Penser par le Diagramme (Paris: Presses Universitaires de Vincennes).

[16] Dahlhaus, C. and Danuser, H. (Eds), 1992, Neues Handbuch der Musikwissenschaft, Vol. 11: Musikalische Interpretation (Laaber: Laaber-Verlag).

[17] Spicker, V., March 1998, Das abstraktmotiv bei Cecil Taylor. MusikTexte, 73/74.

[18] Hatten, R., 2004, Interpreting Musical Gestures, Topics, and Tropes (Bloomington, IN: Indiana University Press).

[19] Schmitt, J.-C., 1990, La Raison des Gestes dans l Occident Médiéval (Paris: Gallimard).

[20] Katsman, R., 2006, Anthropoetic gesture: A key to Milorad Pavić's poetics (Landscape Painted with Tea). Academic Electronic Journal in Slavic Studies, No. 16, University of Toronto.

[21] McNeill, D., 2005, Gesture and Thought (Chicago, IL: University of Chicago Press).

[22] Noll, T., 1998, Wie blättert man in einem hypermedialen Gebärdenlexikon? In: C. Schmauser and T. Noll (Eds.), Körperbewegungen und ihre Bedeutungen (Berlin: Berlin-Verlag Arno Spitz).

[23] Müller, S. and Mazzola, G., 2003, Constraint-based shaping of gestural performance. Proceedings of the ICMC 03 (Ann Arbor, MI: ICMA).

[24] Wieland, R. and Uhde, J., 2002, Forschendes Üben (Kassel: Bärenreiter).

[25] Mazzola, G., 2004, Comprehensive Mathematics for Computer Scientists, Vols I, II (Heidelberg: SpringerVerlag).

[26] Mac Lane, S., 1971, Categories for the Working Mathematician (Heidelberg: Springer-Verlag).

[27] Lages Lima, E., 2003, Fundamental Groups and Covering Spaces (Natick, MA: Peters).

[28] Gabriel, P., 1992, Representations of finite-dimensional algebras. In: A.I. Kostrikin and I.R. Shafarevich (Eds.), Encyclopaedia of Mathematical Sciences, Vol. 73 (Berlin: Springer-Verlag).

[29] Lewin, D., 1990, Klumpenhouwer networks and some isographies that involve them. Music Theory Spectrum, 12.

[30] Serre, J.-P., 1980, Trees (New York: Springer-Verlag).

[31] Lee, J.M., 2000, Introduction to Topological Manifolds (Heidelberg: Springer-Verlag).

[32] Mazzola, G., et al., 1996 ff., RUBATO on the Internet. Available online at: http://www.rubato.org (accessed 26 November 2006).

[33] Benson, D., 1998, Representations and Cohomology, Vol. 1: Basic Representation Theory of Finite Groups and Associative Algebras (Cambridge, UK: Cambridge University Press).

[34] Mazzola, G., 2005, Musical gestures and their diagrammatic logic. Cognitive Science Seminar, AI Lab, Universität Zürich. Available online at: http://www.encyclospace.org/talks/dialog.ppt (accessed 26 November 2006). 